\title{
Mitigation of the Ground Reflection Effect in Real-Time Locating Systems
}

\author{
Dante I. Tapia, Juan F. De Paz, Cristian I. Pinzón, and Javier Bajo
}

\begin{abstract}
Real-Time Locating Systems (RTLS) are one of the most promising applications based on Wireless Sensor Networks and represent a currently growing market. However, accuracy in indoor RTLS is still a problem requiring novel solutions. One of the main challenges is to deal with the problems that arise from the effects of the propagation of radio frequency waves, such as attenuation, diffraction, reflection and scattering. These effects can lead to other undesired problems, such as multipath and the ground reflection effect. This paper presents an innovative mathematical model for improving the accuracy of RTLS, focusing on the mitigation of the ground reflection effect by using Artificial Neural Networks.
\end{abstract}

Keywords: Wireless Sensor Networks, Real-Time Locating Systems, Ground Reflection Effect, Artificial Neural Networks.

\section{Introduction}

Wireless Sensor Networks (WSNs) allow us to obtain information about the environment and act on this, expanding users' capabilities and automating daily actions. One of the most interesting applications for WSNs is Real-Time Locating Systems (RTLS). The most important factors in the locating process are the kind of sensors used and the techniques applied for the calculation of the position based on the information recovered by these sensors. In addition, indoor locating needs still more development, especially with respect to accuracy and low-cost and efficient infrastructures [9] [14]. Therefore, it is necessary to develop Real-Time Locating Systems that allow performing efficient indoor locating in terms of precision and optimization of resources. This optimization of resources includes the reduction of the costs and size of the sensor infrastructure involved on the locating system. In this sense, the use of optimized locating techniques allows obtaining more accurate locations using even fewer sensors and with less computational requirements [9].

\footnotetext{
Dante I. Tapia · Juan F. De Paz · Cristian I. Pinzón · Javier Bajo

Computers and Automation Department, University of Salamanca.

Plaza de la Merced, s/n, 37008, Spain

e-mail: \{dantetapia, fcofds, cristian_ivanp, jbajope\}@usal.es, corchado@usal.es
} 
There are several wireless technologies that may be used by indoor RTLS, such as RFID (Radio Frequency IDentification), Wi-Fi, UWB (Ultra-Wide Band), Bluetooth and ZigBee. However, independently of the technology used, it is necessary to establish mathematical models that allow determining the position of a person or object based on the signals recovered by the sensor infrastructure. Therefore, the position can be calculated by means of several locating techniques, such as signpost, fingerprinting, triangulation, trilateration and multilateration [4] [5]. However, all these techniques must deal with important problems when trying to develop a precise locating system that uses WSNs in its infrastructure, especially for indoor environments.

The electromagnetic waves transmitted and received by the wireless sensor infrastructure used by these systems are affected by some propagation effects, such as reflection, scattering, attenuation and diffraction [3]. Due to these effects, the energy of the transmitted electromagnetic waves is substantially modified between transmitter and receiver antennas in these systems. Thanks to the attenuation effect, it is possible to estimate the distance covered by a wave between a transmitter and a receiver antenna [1]. This is very useful to build RTLS based on these distances, as those based on trilateration [4]. However, reflection, diffraction and scattering effects lead to other problems such as the ground reflection effect [3], a kind of multipath propagation effect. Therefore, it is necessary to define new models and techniques that allow the improvement of accuracy in this kind of systems.

This paper proposes a new mathematical model aimed at improving the precision of RTLS based on wireless sensor networks, especially at indoor environments. This model uses Artificial Neural Networks (ANNs) as the main components to mitigate the ground reflection effect and calculate the position of the elements.

Next, Section 2 explains the problems that the ground reflection effect introduces in RTLS that are based on wireless sensor networks. Section 3 describes a new proposal for reducing the ground reflection effect by using ANNs. Section 4 depicts the experiments performed on a real scenario to validate the accuracy of the new model and also describes the obtained results. Finally, Section 5 presents the conclusions obtained so far and depicts the related future work intended to improve the proposed method, including new applications for it.

\section{Background and Problem Description}

Real-Time Locating Systems based on Wireless Sensor Networks can be seriously affected by some effects related to the electromagnetic waves propagation, especially indoors [9]. Some of these effects are reflection, scattering or attenuation, amongst others. Such effects can provoke which is known as multipath effect, and, more specifically to indoor RTLS based on WSNs, the ground reflection effect [2]. There are several related approaches focused on the study or the mitigation of the multipath or the ground reflection effect [16] [2] [13] [11] [12] [9] [4] [7]. However, these approaches just solve the problem partially and none of them are specifically intended to enhance the performance of Real-Time Locating Systems. 
Real-Time Locating Systems calculate the position of each tag in the system from a set of measurements obtained from the electromagnetic waves transmitted amongst tags and readers (e.g., RSSI or Received Signal Strength Indication), its quality (e.g., LQI or Link Quality Indicator), its Signal to Noise Ratio (SNR) or the Angle of Arrival (AoA) to the reader, amongst many others. In an ideal environment, these measurements would be perfect, with no error or noise, and the calculation of tags positions would be exact. Nevertheless, in the real world, the electromagnetic waves are influenced by effects as reflection, scattering, attenuation and diffraction. Attenuation is, in fact, a desired effect for estimating distances from measurements such as the received power of signals (RSSI). RSSI can be used, indeed, in signpost, fingerprinting and trilateration techniques to estimate distances from signal received power. However, reflection, scattering and diffraction can make the readers to receive additional spurious signals that are undesired copies of the main signal. The reception of such spurious signals makes up the multipath effect. This effect is especially undesired when measuring parameters as the RSSI, the AoA or the TDOA (Time Difference Of Arrival). When the ground is the main responsible of waves reflections, multipath can be modeled as the ground reflection effect, which is described in the next subsection.

\subsection{The Ground Reflection Effect}

The effects that affect the propagation of the electromagnetic waves, such as reflection, scattering, attenuation and diffraction, can reduce or even increase the range of a radio transmission [3]. Specifically, these effects can be a major challenge when designing a RTLS based on WSNs, especially for indoor environments.

The detailed effects of phenomena as attenuation and reflection in the propagation of electromagnetic waves can be calculated by solving Maxwell's equations with some boundary conditions that model the physical characteristics of each object or medium involved [3]. As this calculation can be very complex or even the physical characteristics of each object can be even unknown, there are some approximations to model signal propagation and calculate range transmission. One of these approximations is the ray-tracing technique that simplifies electromagnetic wavefronts to simple particles. Physically, each wavefront is the locus of spatial points presenting the same phase for a certain electromagnetic wave. In the ray-tracing technique, each wavefront is considered to be a particle traveling from the transmitter to the receiver antennas. This is very useful to model reflection and refraction effects, although it ignores the scattering phenomenon [3]. An electromagnetic wave transmitted by a certain wireless source will be reflected, diffracted or even scattered by the multiple objects placed throughout the environment. This way, the antenna of the destination node will receive undesired copies of the transmitted signal. Even worse, these additional signals will be possibly delayed in time and shifted in frequency and phase. When a single ground reflection effect predominates in the multipath effect, a two-ray model, as shown in Figure 1, can be used. 


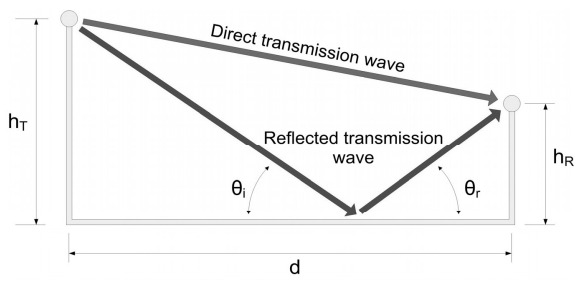

Fig. 1 Graphical representation of the ground reflection effect. Direct and reflected transmission waves travel from the transmitter to the receiver antennas, causing to be constructively or destructively added due to phases difference.

\section{Mitigation of the Ground Reflection Effect}

In ideal conditions, the modeling of the relationship between RSSI levels and distances between antennas has a decaying exponential shape. Nevertheless, when ground reflection effect is taken into account, the process of approximation of the relationship between the RSSI levels and the distances between antennas is complex and problematic. Therefore, it is necessary to use other models that allow considering the ground reflection effect in order to obtain a reliable estimation of the distances between tags and readers.

The model presented in this paper proposes the use of two Multi-Layer Perceptron [10] artificial neural networks to improve the precision of RTLS. On the one hand, the first MLP allows mitigating the ground reflection effect when estimating distances from power signal levels used to calculate the positions of users and objects by different locating techniques. On the other hand, the second MLP calculates the final positions of users and objects in the environment, using the output of the first MLP and acting, indeed, as a new locating technique that improves the precision of other compared techniques

For a certain range of RSSI values, there are fluctuations in the distance values regarding the RSSI levels. Thus, a certain RSSI value can mean distinct distances. In order to model the ground reflection effect we utilize time series applied to the first Multi-Layer Perceptron. Artificial Neural Networks allow forecasting a value according to the received historical values. Therefore, in this work the neural network is provided as inputs both the current detected RSSI value and the RSSI values detected in previous time instants. The neural network is made up of $n$ input neurons, being $n$ the time instants taken into account: $t, t-1, \ldots, t-(n-1)$. The intermediate layer of the neural network is configured following the Kolmogorov theorem [6] and choosing $2 n+1$ neurons.

In order to improve the forecast of the time series it was opted to incorporate the RSSI levels provided by other readers into the neural network. This way, the distances forecasting is done using a subset of the deployed readers in the system simultaneously. The neural network has $k$ input groups with $n$ neurons each of them. These $n$ neurons correspond with the $n$ values of the time series. Likewise, the $k$ groups correspond with number of readers that are considered for the distance estimation. This number of readers is set in advance, thus selecting the 
readers with highest measured RSSI levels from the tag. The intermediate layer is made up of $2(k+n)+1$ neurons, whereas output layer is formed by $k$ neurons (i.e., a neuron per each reader). The groups of input neurons are ordered according to the current RSSI level from highest to lowest. Therefore, the first output of the neurons is associated to the reader that received the highest RSSI level and so on.

Our proposed model captures data from the estimation of the positions by the trilateration algorithm. It stores these in a memory to subsequently use them to carry out the training of the second MLP. This way, this second MLP allows us to make the fastest estimations and is more responsive to variations in the distances resulting from the reflections of the waves emitted. Input data in the second MLP corresponds with the distances calculated by means of the first MLP from a prefixed number of readers and the position of the readers. These readers are selected according to the lowest distances they have to the tag. Output has two coordinates, one for each space coordinate. The number of neurons in the hidden layer is $2 n+$ 1 , where $n$ is the number of neurons in the input layer. Finally, there is one neuron in the output layer. The activation function selected for the different layers has been the sigmoid. Furthermore, the neurons exiting from the hidden layer of the neural network contain sigmoidal neurons. Network training is carried out through the error backpropagation algorithm [7].

\section{Experiments and Results}

In order to test the performance of this model into an indoor environment, we proceeded to deploy a WSN infrastructure made up of several ZigBee nodes (i.e., readers and tags). These devices, called n-Core Sirius A for readers and Sirius B for tags, have both $2.4 \mathrm{GHz}$ and $868 / 915 \mathrm{MHz}$ versions and have several communication ports to connect to distinct devices, including a wide range of sensors and actuators. n-Core devices form part of the n-Core platform, which offers a complete API (Application Programming Interface) to access all its functionalities [8]. The ZigBee network was formed by 15 fixed Sirius A nodes acting as readers and distributed throughout three rooms. The total size of the monitored area was $19 \mathrm{~m}$ per $19 \mathrm{~m}$. The distribution of the readers was done in this way so that each tag could be identified by several readers simultaneously. Therefore, the selected locating techniques (i.e., signpost, fingerprinting and trilateration) could be applied using several simultaneous measurements. Firstly, as a previous step before the estimation of the tags positions, it was carried out the training of the neural network built to estimate the distances between nodes from the RSSI levels. A test tag was successively moved through different predefined location sequences (i.e., zones inside the laboratory). This way, it was calculated the relationship of the measured RSSI levels with the real distances between the tag and the readers. For doing this, it was measured the detected RSSI levels between the tag and each of the 15 readers. Thus, the RSSI-distances measurements were used to make predictions in the time series. In total, 200 cases were generated for the training of the neural network. In addition, it was randomly chosen different positions throughout the zones to generate 100 new cases and estimate each position by means of both the neural network and other approximation methods to compare them. These other methods 
were SVR (Support Vector Regression) [15], a linear regression model and a logarithmic regression model. The calculation of the relationship between the RSSI levels and the distances in the training data set is necessary because the characteristics of the existing materials affect considerably to the detected distances.

As expected, the neural network obtained better results than SVR, the linear regression model and the logarithmic model because it presents a lower error for the distances estimation. The regression model obtained for the logarithmic regression fits in a very high grade the training data, as this model obtains an $R^{2}=0.9907$. Likewise, the linear regression model obtains an $R^{2}=0.8968$, which is also a high value. Basing on these $R^{2}$ values, both models can be considered as valid for the estimation. That is, the estimations made are significant and any other method that improves these results would also be valid. The errors for the MLP are lower than for the rest of the compared methods. Moreover, for the MLP the errors are concentrated in a certain range of RSSI levels. This allows creating reliable values outside some determined frequency ranges. Analyzing the dispersion of the error for each the compared model, shown in the Figure 2, it can be seen that the MLP offers the lowest dispersion and does not present so extreme values as SVR and linear regression do. Figure 2 (left) shows the box plot diagrams for the SVR, the regression models and the MLP. As can be seen, the MLP presents the lowest data variance and the minimum error.
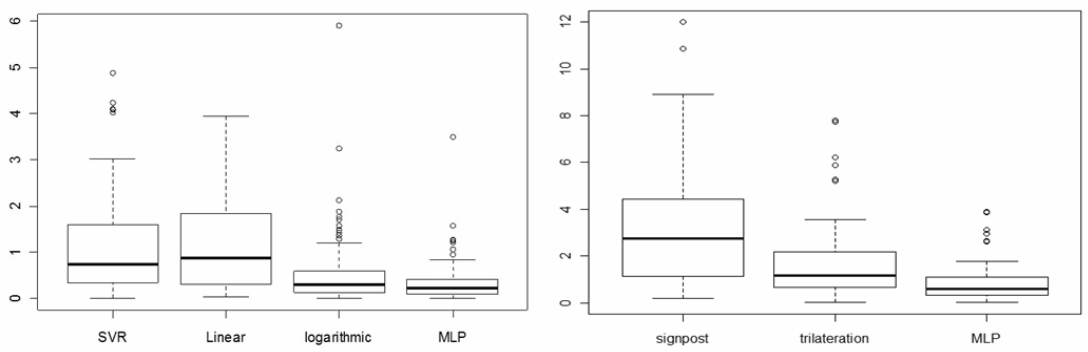

Fig. 2 Box plots representing the absolute error for the RSSI-distances relationship when using the different approximations (left) and the location errors for the different compared locating techniques (right).

The box plots representing the error information are presented in Figure 2 (right). As can be seen in the figure, the MLP provides lower estimation errors than the signpost and trilateration by themselves, that is, without modeling the RSSI and position behaviors.

\section{Conclusions and Future Work}

Amongst the wide range of Wireless Sensor Networks applications, Real-Time Locating Systems are emerging as one of the most exciting research areas. However, the operation of RTLS can be affected by undesired phenomena as the multipath effect, and more specifically, the ground reflection effect. 
This paper proposes a new mathematical model aimed at improving the precision of WSN-based RTLS. The use of measurements from several readers as inputs of the MLP in the proposed model reduces even more the prediction error. This way, the ground reflection effect is mitigated and the approximations provided by other methods with high adjustment goodness, as the logarithmic regression model, were improved. This improvement in the distances forecasting is very relevant to estimate the positions of the tags, thus optimizing the overall calculations of locating techniques. In addition, the neural network responsible for calculating the final position reduces the error level of traditional methods from the information provided by them. The results obtained demonstrate that the use of ANNs allows improving the approximations provided by the locating techniques.

As future work it is planned the reduction of the readers necessary to perform the locating process, as well as the implementation in larger environments. Future work also includes the study of more detailed multipath models as Ricean and Rayleigh fading or shadowing [13].

Acknowledgments. This work has been supported by Spanish Ministry of Science and Innovation Project Ref. TRA2009_0096.

\section{References}

1. Barclay, L.W., I.O.E. Engineers.: Propagation of Radiowaves. Iet (2003)

2. Kim, E.S., Kim, J.I., Kang, I.-S., Park, C.G., Lee, J.G.: Simulation Results of Ranging Performance in Two-Ray Multipath Model. In: International Conference on Control, Automation and Systems, ICCAS 2008, pp. 734-737 (2008)

3. Goldsmith, A.: Wireless Communications. Cambridge University Press, Cambridge (2005)

4. Liu, H., Darabi, H., Banerjee, P., Liu, J.: Survey of Wireless Indoor Positioning Techniques and Systems. IEEE Transactions On Systems, Man, and Cybernetics, Part C: Applications and Reviews 37(6), 1067-1080 (2007)

5. Kaemarungsi, K., Krishnamurthy, P.: Modeling Of Indoor Positioning Systems Based On Location Fingerprinting. In: Twenty-Third Annual Joint Conference of the IEEE Computer and Communications Societies, INFOCOM 2004, vol. 2, pp. 1012-1022 (2004)

6. Katsuura, H., Sprecher, D.: Computational Aspects of Kolmogorov's Superposition Theorem. Neural Networks 7(3), 455-461 (1994)

7. Lecun, Y., Bottou, L., Orr, G.B., Müller, K.R.: Efficient Backprop. LNCS, pp. 5-50. Springer, Heidelberg (1998)

8. N-Core, N-Core: A Faster and Easier Way to Create Wireless Sensor Networks (2010), http: / /Www. N-Core. Info (retrieved October 27, 2010)

9. Nerguizian, C., Despins, C., Affès, S.: Indoor Geolocation with Received Signal Strength Fingerprinting Technique and Neural Networks. In: de Souza, J.N., Dini, P., Lorenz, P. (eds.) ICT 2004. LNCS, vol. 3124, pp. 866-875. Springer, Heidelberg (2004)

10. Nguyen, H., Chan, C.: Multiple Neural Networks for a Long Term Time Series Forecast. Neural Computing \& Applications 13(1), 90-98 (2004) 
11. Ray, J.K., Cannon, M.E., Fenton, P.C.: Mitigation Of Static Carrier-Phase Multipath Effects Using Multiple Closely Spaced Antennas. Navigation-Washington 46(3), 193-202 (1999)

12. Salcic, Z., Chan, E.: Mobile Station Positioning Using GSM Cellular Phone and Artificial Neural Networks. Wireless Personal Communications 14(3), 235-254 (2000)

13. Schmitz, A., Wenig, M.: The Effect of the Radio Wave Propagation Model in Mobile Ad Hoc Networks. In: Proceedings of the 9th ACM International Symposium on Modeling Analysis and Simulation of Wireless and Mobile Systems, Terromolinos, Spain, pp. 61-67 (2006)

14. Tapia, D.I., De Paz, J.F., Rodríguez, S., Bajo, J., Corchado, J.M.: Multi-Agent System For Security Control On Industrial Environments. International Transactions on System Science and Applications Journal 4(3), 222-226 (2008)

15. Vapnik, V.N.: Statistical Learning Theory. Wiley Interscience, Hoboken (1998)

16. Xie, J.J., Palmer, R., Wild, D.: Multipath Mitigation Technique in RF Ranging. In: Canadian Conference on Electrical and Computer Engineering, pp. 2139-2142 (2005) 